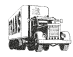

TRANS $\cdot$ núm. $23 \cdot 2019$

DOSIER · 51-66

El nombramiento de 204 traductores e intérpretes soviéticos llevado a cabo por el Servicio de Inteligencia Militar del Ejército Rojo constituye un medio importante para la institucionalización de la traducción e interpretación en la guerra civil española. Entendemos el concepto de institucionalización de la traducción como una forma de legitimación jurídico-administrativa, social y moral de la actuación profesional de los traductores e intérpretes, que por un lado favorece y por otro obstruye la acción de la traducción como práctica cultural y social. En este artículo se analizan los mecanismos de institucionalización y su efecto en la actuación de los traductores e intérpretes.

PALABRAS CLAVES: institucionalización, traducción e interpretación, conflicto, ideología.

\title{
La traducción e interpretación institucionalizadas en la guerra civil española
}

IRYNA ORLOVA

Universidad de Graz
Institutionalized translation and interpretation in the Spanish Civil War

The appointment of 204 translators and interpreters to serve in the context of the Spanish Civil War carried out by the Military Intelligence Service of the Red Army constitutes an important measure for the institutionalization of translation in this context. The concept of institutionalization of translation refers to a form of legal-administrative, social, and moral legitimation of the professional performance of translators and interpreters, which, on the one hand, favors and, on the other hand, obstructs the action of translation as a cultural and social practice. The article analyzes the mechanisms of institutionalization and their effect on the performance of translators and interpreters during the war.

KEY WORDS: institutionalization, translation and interpretation, conflict, ideology. 


\section{INTRODUCCIÓN}

La guerra civil española (I936 a r939) fue un 52 conflicto político y social que excedió los límites territoriales del país y atrajo la atención de las potencias mundiales en el nivel institucional, así como de numerosos voluntarios de forma personal. El contingente extranjero en territorio español presentó una notable heterogeneidad cultural y lingüística, que llegó a agrupar a ciudadanos de 53 naciones en las Brigadas Internacionales. Este hecho produjo una situación sin precedente de multilingüismo y un incremento de la demanda de todo tipo de mediadores. La comunicación y la traducción e interpretación entre los y las voluntarias extranjeras en España es el tema central del proyecto «Traducción e interpretación en la Guerra Civil» (Universidad de Graz) $)^{\mathrm{r}}$, en el que se enmarca este artículo.

Al conocer el apoyo activo de Alemania e Italia a los sublevados, el gobierno de la Unión Soviética cambia el curso inicial de la no intervención y aprueba entre mediados de agosto y finales de septiembre de 1936 (Miralles, 2009: 2o) la asíllamada Operación $X$. Esta operación la dirige el GRU (Servicio de Inteligencia Militar del Ejército Rojo) y consiste en el suministro de armas y transporte militar, así como en labores de asesoría militar. Unos 600 asesores en diferentes áreas militares son enviados directamente a la guerra (Miralles, 2009: 35). Estos asesores, según el plan inicial, tienen que ir acompañados cada uno por un traductor e intérprete personal.

I Proyecto de investigación «Traducción e interpretación en la Guerra Civil» («Dolmetschen und Übersetzen im Spanischen Bürgerkrieg, 1936-1939») en la Universidad de Graz (Instituto de Traductología), financiado por el Jubiläumsfonds der Oesterreichischen Nationalbank (OeNB). https://translationswissenschaft.uni-graz.at/ de/forschen/forschungsprojekte/dolmetschen-unduebersetzen-im-spanischen-buergerkrieg-I936-I939/ projektbeschreibung/
Sin embargo, su número no llega a superar los 204 (según el Informe sobre el número de militares soviéticos enviados a España en comisión de servicio RGVA, F.33987. I.3.A.II43. P.I27, citado en Rybalkin, 2009: 8I).

El grupo de traductores e intérpretes enviado por el GRU, su actuación en diferentes situaciones y su función en el transcurso de la guerra constituyen el objeto de estudio del presente artículo. Su nombramiento militar oficial por una institución estatal les atribuye características significativas que influyen en su práctica profesional como traductores e intérpretes militares y en la institucionalización de la traducción e interpretación en la guerra civil. Este estudio se centra en el proceso, los mecanismos y el funcionamiento de la institucionalización de la traducción e interpretación en condiciones de hostilidad política e ideológica.

A pesar de la enorme cantidad de trabajos dedicados al tema de la guerra civil española, han recibido poca atención el aspecto de la traducción e interpretación y otras formas de mediación lingüística o comunicación multilingüe. La información aislada sobre la comunicación dentro de las Brigadas Internacionales se puede encontrar dispersa en algunos estudios históricos sobre la guerra civil española (Kowalsky, 2004; Brigadistas, 2005; Kirschenbaum, 20I8). Desde el punto de vista traductológico se enfoca el tema en los trabajos de Baigorri (2012), Rodríguez-Espinosa (2016) y Wolf (20I7). Baigorri (20I2) abre el discurso científico sobre la interpretación en la guerra civil española y hace una tipología generaliza$\mathrm{da}$ «de la procedencia sociológica de quienes actuaron como traductores e intérpretes» (Baigorri, 2012: 103). E1 autor menciona algunos aspectos de ética de la interpretación (como, por ejemplo, la lealtad de los y las traductoras e intérpretes) y señala la variedad de funciones 
que cumplían las y los intérpretes en la guerra. Rodríguez-Espinosa (2016) traza las biografías de algunas traductoras e intérpretes cuya labor está relacionada con los servicios de seguridad. En su artículo la traducción e interpretación se enmarcan en un contexto de ideologías y políticas soterradas que guían la actuación de los individuos según patrones convencionales. Wolf (20I7) señala una nueva dimensión cultural y lingüística del conflicto bélico en España, que se abre a través del análisis de situaciones de comunicación multilingüe, la traducción y la interpretación, y el carácter constitutivo de estas prácticas para el transcurso de la guerra.

Las preguntas que se plantean en este artículo se refieren a los mecanismos de institucionalización, a las instituciones autorizadas para ejercerla, al proceso de la selección de un grupo profesional y a los efectos de institucionalización en la actuación de los traductores e intérpretes. Estos aspectos se analizan dentro del marco teórico de la sociología de la traducción (Inghilleri, 2005; Wolf/Fukari, 2007; Inghilleri/ Harding, 2010) y se basan en una evaluación sistemática de un corpus exhaustivo de fuentes.

Los y las intérpretes enviados desde la Unión Soviética presentan unas características distintivas que permiten considerarlos como un grupo aparte. En las Brigadas Internacionales no existe un proceso de selección unitario sino que se opta por aquellas personas que pudieran demostrar un cierto conocimiento de los idiomas (Baigorri, 2012: 104); la demanda se cubre, sobre todo, de manera espontánea o $a d$ hoc con los medios disponibles (Baigorri, 20I2: 88) y nunca son oficialmente nombrados traductores e intérpretes. No es este el caso de los y las mediadores profesionales enviados por el GRU. Este grupo sí que fue seleccionado por una comisión militar, cada uno recibió el nombramiento militar, fueron trasladados a España, tenían un estatus oficial y a veces disfrutaban de poderes más amplios que los asignados comúnmente a un traductor o intérprete. Estas razones nos llevan a considerar el factor de la institucionalización de la traducción e interpretación por parte del Ejército Rojo como un mecanismo crucial para el análisis de contextos marcados tanto por las hostilidades políticas como por la heterogeneidad cultural y lingüística.

\section{MARCO TEÓRICO Y METODOLOGÍA DEL ESTUDIO}

El concepto de la traducción institucionalizada se ha desarrollado en los últimos años en el marco de los enfoques sociológicos y culturales de la traductología. El primer argumento a favor de la traducción como institución lo define Hermans (1995) cuando subraya la «cara pública» de la traducción: su presencia en la vida cotidiana, su codificación en diccionarios, su representación en el nivel profesional (traductores e intérpretes) y organizativo (asociaciones de traductores e intérpretes) y en centros de formación profesional (Hermans, 1995: 5). Además, es significativo, según este autor, la cantidad de agentes involucrados en la traducción, como clientes, traductores e intérpretes, agencias, editoriales, lectores, cuyas expectativas generan una serie de normas y convenciones de traducción (Hermans, 1995: 9).

En el presente artículo entendemos el concepto de institucionalización como «human activity that installs, adapts, and changes rules and procedures in both social and political spheres» (Keman, 20I7, web). Distinguimos la traducción e interpretación institucionalizada de la traducción e interpretación institucional, que se define como «translation that occurs in institutional settings» (Ji-Hae, I998: I4I) (por ejemplo, en el Parlamento Europeo). La tra-

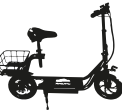

53 
ducción e interpretación institucionalizada, en cambio, no se limita solo al marco de las normas de la institución (u organización) en la que se realiza, sino que se desarrolla como una práctica cultural dentro de una comunidad. Sus características distintivas son: la presencia de una persona mediadora, la participación de múltiples agentes que realizan una variedad de acciones, la negociación de las normas (que luego pueden adquirir un soporte normativo procedente de una organización) y una interacción con otros campos de la vida social. Estos aspectos son clave para el análisis de la institucionalización de la traducción e interpretación en la guerra civil, donde hubo también otras formas de comunicación y traducción e interpretación intercultural.

Los mecanismos de la institucionalización de la traducción e interpretación en la guerra civil española se enfocan en el presente artículo desde la teoría sociológica de Pierre Bourdieu. Esta perspectiva permite abordar el tema tomando en consideración la relación entre el individuo y la estructura de jerarquías sociales, el funcionamiento de los capitales (Bourdieu, I986: 246) y la actuación del individuo bajo unas condiciones determinadas. Además, se analiza la legitimación y el funcionamiento de las instituciones como factores constituyentes de las prácticas sociales y culturales.

Para analizar los factores que definen la posición del grupo soviético de intérpretes en la guerra nos orientamos al concepto de «capital» de Bourdieu (I986). Se definen cuatro tipos de capitales (económico, cultural, social y simbólico); entre ellos, el cultural y el social son los que influyen en mayor grado en la posición de los traductores e intérpretes en la guerra. El capital cultural se manifiesta en el bagaje de conocimientos culturales que poseemos, p.ej. los conocimientos generales sobre el mundo, la situación política o la geografía de un país. Una forma de capital cultural es el capital lingüístico, que es de particular relevancia para la traducción e interpretación (Bourdieu, I986: 243). E1 capital lingüístico se define como el poder para actuar sobre los mecanismos de formación de los precios lingüísticos, para hacer que funcionen en provecho propio las leyes de formación de los precios y así recoger la plusvalía específica (Bourdieu, I993: 80). Para Bourdieu, quien tiene la palabra, quien posee el monopolio de comunicar y comprender, impone por completo la arbitrariedad de sus intereses. El capital social remite a la posesión de una red duradera de relaciones más o menos institucionalizadas de reconocimiento mutuo y a la adhesión a un grupo (Bourdieu, 1986: 248).

La suma de los capitales produce efectos sociales que tienen su base en el poder. La posición del actor en el campo depende del volumen y estructura del capital que posee, así como de los principios de diferenciación social propios del campo:

Thus the perception of the social world is the product of a double structuring: on the objective side, it is socially structured because the properties attributed to agents or institutions present themselves in combinations that have very unequal probabilities [....] On the subjective side, it is structured because the schemes of perception and appreciation, especially those inscribed in language itself, express the state of relations of symbolic power (Bourdieu, 1989: 20).

Es decir, cada campo posee su propio sistema de clasificación y valoración de capitales. El valor definitivo del capital que uno posee y su posición resulta de su reconocimiento en un campo concreto o del capital simbólico. La lucha por el capital simbólico también tiene lugar en dos niveles: en el nivel objetivo o de representación, 
por medio de la demostración de ciertas realidades, o en el nivel subjetivo o de percepción, por medio del cambio en las categorías de clasificación y valoración del mundo (Bourdieu, I989: 20). La segunda opción, «which is a struggle to impose the legitimate principle of vision and division» (Bourdieu, I989: 2I), es frecuente en la lucha política. Visto desde esta perspectiva, el análisis de la institucionalización de la traducción en la guerra arroja luz sobre el funcionamiento de la traducción e interpretación como instrumento político, sociológico, cultural e ideológico.

E1 poder de legitimar ciertos comportamientos, según Bourdieu (1989), pertenece a las instituciones que han ganado y acumulado suficiente autoridad y poder simbólico en la lucha previa:

The power to impose upon other minds a vision, old or new, of social divisions depends on the social authority acquired in previous struggles. [...] In this way, the power of constitution, a power to make a new group, through mobilization, or to make it exist by proxy, by speaking on its behalf as an authorized spokesperson, can be obtained only as the outcome of a long process of institutionalization, at the end of which a representative is instituted, who receives from the group the power to make the group (Bourdieu, I989: 23).

En resumen, mediante la institucionalización se produce la elaboración, legitimación e imposición de valores y comportamientos sociales que tienen su origen en una de las partes que participan en el conflicto. Basándonos en la teoría de Bourdieu, definimos y analizamos los agentes que organizaron y coordinaron estos procesos de institucionalización. Asimismo, nos centramos en sus mecanismos y efectos en relación con la traducción e interpretación en la guerra. El estudio de estos procesos nos permite acercarnos a las implicaciones ideológicas de la traducción como actividad fundamental en los conflictos bélicos multinacionales.

$\mathrm{El}$ corpus de análisis lo componen en primer lugar las biografías de los y las intérpretes de la URSS recién publicados en libro Iz Moskvy - v stranu «Iks». Kniga pamjati sovetskih dobrovol'cev - ǔastnikov Graždanskoj vojny v Ispanii 1936I939 gg. (Arcybašev, 2015, T. 2: 294-343). La edición se basa en la «Lista del mando, oficiales y soldados del Ejército Rojo y de otras personas participantes en la guerra civil española de 1936 a I939", redactada en el Estado Mayor de las Fuerzas Armadas Soviéticas en los años 6o (Arcybašev, 2015: I2, T. 2: 294-343). El libro contiene una lista de 198 personas «enviadas con la misión de traductor e intérprete». En una lista aparte se mencionan 8 personas «enviadas con la misión de traductor e intérprete, pero que nunca llegaron a España». Encontramos en este libro a 5 ciudadanos soviéticos enviados por el GRU como operadores de radio (Valentina Aleksandrovskaja, Vasilij Jabločnikov), cifradoras (Evgenija Tarabanova) o con una misión especial (Georgij Klark, Ahmed Latypov), pero que también ejercieron como intérpretes. De la misma forma, los periodistas Mihail Kol'cov y Elizaveta Kol'cova traducen e interpretan para sus compañeros en muchas ocasiones.

Es preciso señalar que hay otros grupos de traductores e intérpretes que trabajan para el Ejército Rojo, pero que por diversas razones no forman parte del corpus analizado en este artículo. Un primer grupo lo componen personas de nacionalidad española con conocimientos de lenguas. Los datos de estas personas son muy escasos y no son objeto de análisis en este trabajo. Otros grupos que trabajan para los militares soviéticos son los formados por voluntarios de otros países de lengua eslava o de los países bálticos (p.ej. la polaca Lidija Kuper, el letón Ben-

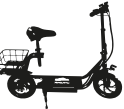

55 


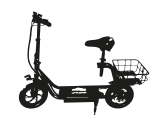

56 jamin Kur) y los «rusos blancos» residentes en otros países como Francia, España, EE.UU. que participan como voluntarios en la guerra civil española (p.ej. Aleksej Èjsner, Pavel Naumenko, Constant Alexis Brusiloff [Nigehorodzeff], Mihail Krigin, Julija Rodríguez-Danilevskaja, Elena Rodríguez-Danilevskaja). Estos grupos de los y las traductores e intérpretes los trataremos aparte.

Otra parte del corpus lo componen las memorias autobiográficas de los voluntarios y las voluntarias soviéticos que participaron en la guerra civil. Para el análisis de estas fuentes nos orientamos hacia la metodología propia del análisis estructural de contenido. En primer lugar, de los textos mencionados, seleccionamos los pasajes que se refieren a la comunicación o interpretación y traducción en la guerra. Todos estos pasajes se codifican con conceptos propios de la traductología, como por ejemplo Conocimientos de lengua, Problemas de comunicación, Modo de interpretación, Situación de interpretación, etc. Entre estos conceptos distinguimos también la Interpretación institucionalizada. Codificar los pasajes permite reunir los que están marcados con este concepto para su subsiguiente análisis. Este análisis se realiza a partir de las preguntas de investigación formuladas en el estudio. Los resultados son sintetizados e interpretados a la luz de la teoría sociológica de la traducción.

\section{LOS AGENTES DE LA INSTITUCIONALIZACIÓN}

Los primeros brigadistas llegan a Albacete el I4 de octubre de I936, donde se organiza un centro de reclutamiento de voluntarios. Albacete fue elegida como encrucijada para la organización y para el posterior traslado de dichos voluntarios a los lugares donde su labor fuera necesaria
(Brigadistas, 2005: 133). La primera impresión de los voluntarios al cruzar la frontera francesa y al llegar a Albacete es la confusión de lenguas y una aglomeración de gente de diferentes nacionalidades: «Fue una Babel de todas las lenguas posibles. Nunca he visto algo tan extraordinario - una hermandad increíble de diferentes nacionalidades» (Dufek, 2008, web; cita traducida al español del checo).

Muy pronto, el multilingüismo de las Brigadas Internacionales empieza a ser problemático, en especial en lo que afecta a cuestiones organizativas y de mando en las acciones militares. Así lo comenta el comandante de la XI Brigada Internacional Emilio Kleber, apodo de Manfred Stern:

De momento han sido registradas aproximadamente veinte nacionalidades. Como consecuencia tenemos unas dificultades enormes. Yo, además del nativo alemán y ya casi nativo ruso, hablo español y francés... de ser sincero hablo español muy mal. Pero aun así es insuficiente. ¿Cómo puedo hablar con un griego que además solo habla turco? Es sabido que la construcción de la Torre de Babel fracasó a causa de una mezcla de lenguas. Aquí tenemos una mezcla igual de grande. Solo hay una solución: organizar las compañías, o mejor, batallones, según las lenguas (Èjsner, ı986, web; cita traducida al español del ruso).

La idea de separación lingüística de las Brigadas Internacionales no es apoyada de manera unánime. Algunos, como por ejemplo el general Lukacs, apodo de Máté Zalka, comandante de la XII Brigada Internacional, considera esta medida como una estrategia para debilitar o reforzar algunos batallones:

¿Por qué no ha de venir aquí para enterarse de nuestras dificultades sobre el terreno? Y ¿qué lógica es esa? Separa dos batallones franceses 
y une dos alemanes. ¿Por qué? Tampoco se tiene en cuenta que el noventa por ciento de los italianos vivían en Francia y hablan francés, mejor o peor. ¿Por qué no los ponen en una brigada con los franceses y los belgas? [...] Lo que quiere Kléber no es aliviar la situación, sino reforzar su brigada a expensas de la nuestra (Eisner, 1972: 50).

En el contraste de las dos opiniones de estos comandantes ya se cristaliza la problemática lingüística que acompaña al conflicto bélico y político. El problema del multilingüismo se resuelve en diferentes áreas del ejército republicano de maneras diferentes: «español de trinchera», lingua franca (español, francés, alemán, yidis o esperanto, etc.) o los intérpretes (Wolf, 20I7: 158-I67). El uso y la forma que adopta cada una de las prácticas lingüísticas no son arbitrarios y muestran una clara relación con causas políticas e ideológicas.

En la Unión Soviética el órgano principal de reclutamiento de traductores e intérpretes es el GRU, una sección de la Inteligencia Militar del Ejército Rojo. Desde I929, el Ejército Rojo reconoce oficialmente el título de «intérprete militar», que prevé un soporte jurídico-normativo para el desempeño de la profesión de intérprete (Orden del Comisariado Militar de la Unión Soviética No I25 del 2I de mayo de I929 «Sobre la institución del título para los rangos altos del Ejército Rojo de "Intérprete militar"»). El GRU se hace responsable de la selección, el envío y la coordinación del grupo soviético de intérpretes para la guerra civil. Ellos van acompañando a los asesores, enviados por el Ejército Rojo y que tienen una presencia activa en el Estado Mayor del Ejército, en el Ministerio de la Guerra, así como en las Brigadas Internacionales (Miralles, 2009: 3I). El ratio de los y las intérpretes de la URSS no es de uno a uno con los asesores. Sin embargo, con la plantilla de asesores, traducto- res e intérpretes el GRU cubre los puntos clave de coordinación y mando en la guerra.

La institucionalización de la traducción e interpretación en la guerra supone una etapa de la selección de personal. El reclutamiento se realiza a través de los puestos de trabajo o de centros educativos donde los candidatos y candidatas habían presentado primero sus solicitudes para ser enviados a España. Muchos autores subrayan en sus memorias el carácter voluntario de las solicitudes y las ansias de ir a España:

[...] nosotros jóvenes estudiantes, al igual que todos los soviéticos, soñábamos con ir a España (Abramson, 2002: 215);

[...] inesperadamente se cumple el sueño: me invitan al despacho del director de Intourist que me propone ir a España. Con ganas acepto la propuesta (Pokrovskaja, I989: 48; cita traducida al español del ruso).

Las primeras personas seleccionadas para el grupo de intérpretes normalmente tienen un título universitario y trabajan en organizaciones de renombre. La Comintern ya tiene una práctica propia de traducción entre sus secciones y representaciones por todo el mundo. La extendida red internacional es coordinada por personas con conocimientos de lenguas que ejercen de referentes para España, Francia o países de América Latina, traductores, redactores y secretarios (Sof ja Aleksandrovskaja, Kobyljanskij Kazimir, Fëdor Kravčenko, Elena Lebedeva, Lopes Markučči, Natal'ja Ustromskaja). Además, la Escuela Internacional Lenin (EIL), fundada en Moscú como centro educativo de la Comintern y destinada a la formación en la doctrina marxista de los jóvenes funcionarios de los Partidos Comunistas de todo el mundo, tiene sus propios traductores e intérpretes que coordinan los Sectores nacionales de la EIL

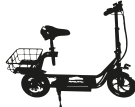


(Elizaveta Gricevskaja, Ljudmila Ivanickaja, Marija Polano-Piras, Julija Rozinger). Otras organizaciones y traductores involucrados son (Arcybašev, 2015, T. 2: 294-343):

- Agencia Estatal de Viajes «Intourist»: Aleksandra Bahmutskaja, Tat'jana Belaja, Semën Gankin, Genrietta Grinberg, Elena Konstantinovskaja, Ljubov' Meston, Elizaveta Mihajlova, Evgenija Oliver, Anna Petrova, Aleksandra Švarc, Irina Vitkovskaja, etc.;

- Redacciones de revistas o periódicos: Paulina Abramson, Benjamin Abramson, Isidor Lasker, Mihail Kol'cov, Vol'f Zlotnickij, etc.

- Ministerios de la Unión Soviética (o Comisariados de Pueblo): Juan Acosta, Avrora Bessmertnaja, Marija Fortus, Nikolaj Fel'dblum, Pavel Liminik, Boris Zil'berg, etc.

Más tarde son reclutados estudiantes de los siguientes centros educativos (Arcybašev, 2015, T. 2: 294-343):

- Instituto del Turismo Extranjero (Leningrado): Elena Aver janova, Semën Bank, Roza Borovskaja, Nina Butyrina, Mark Gankin, Izrail' Gimzel'berg, Haim Gluzman, Raisa Zagadskaja, etc.,

- Universidad Estatal de Leningrado: Èsfir Alenikova, Judit Brill', Sof ja Fridman, Elena Feerštejn, Leon Gromov, Krever Ėlizar, Georgij Kjalin, etc.,

- Instituto Narimanov de Ciencias Orientales (Moscú): Nikolaj Anan'in, Nikolaj Andreev, Avetik Badaljan, Tat' jana Ivanova, etc.,

- Instituto Pedagógico de Lenguas Extranjeras (Moscú): Zinaida Antonjuk, Viktorija Vajnštok, Stepan Vorob'ëv, Ksenija Zajčenko, etcétera.

Las personas seleccionadas en estos centros van a Moscú al Departamento Central de
Inteligencia, donde les espera una entrevista con Gaj Tumanjan, Jefe de la Sección secreta «A». Al llegar a España se presentan ante Jan Berzin, Consejero Jefe militar de las tropas republicanas y Aleksandr Orlov, Jefe del NKVD, Comisariado del Pueblo para Asuntos Internos. Los criterios de selección son variados. Los voluntarios y las voluntarias militan (o han militado) en el Komsomol (organización juvenil del Partido Comunista) o en el Partido Comunista y tienen un buen expediente personal. Están en una buena condición física y han pasado el control médico: algunos son rechazados por tener enfermedades cardiovasculares (Zernova, I998: 138). El nivel profesional, es decir, el conocimiento del español y la experiencia en traducción van descendiendo con el tiempo: primero, van personas con experiencia y conocedoras de la lengua española, luego, estudiantes de lenguas románicas, y al final, estudiantes de otras carreras. El capital social es una gran ventaja: se da preferencia a la gente que tiene contactos con extranjeros o contactos universitarios. Vista la cantidad de mujeres entre los traductores e intérpretes, el género no es un criterio discriminatorio en la selección. Sin embargo, ciertos testimonios apuntan a que en los últimos meses de la guerra ya no se admite a mujeres (Pricker, I989: 373). Tampoco son obstáculos la nacionalidad o el hecho de tener hijos.

El proceso de institucionalización de la traducción e interpretación sigue con la creación de dos centros de formación de traductores e intérpretes: uno en Leningrado de 3 meses en la sede de la Universidad Estatal de Leningrado (Zernova, I998:I35), y el otro, de doce días, en la zona republicana en Caldetas (Mihail Ivanov citado en Abramson, 2002: 215). Las organizadoras de los cursos son las intérpretes Elena Konstantinovskaja, Anna Petrova, Aleksandra Švarc (Leningrado) y Olga Filippova y Ljusija 
Pokrovskaja (Caldetas). En España, el curso se organiza bajo la orden del GRU, como recuerda Ljusija Pokrovskaja:

De Moscú ha venido un grupo de chicasestudiantes de universidades diferentes. En su mayoría son de Filología francesa. La tarea es enseñarles español, para que puedan empezar a trabajar lo antes posible. A mí me nombran profesora, pero sin abandonar las tareas directas de traductora. [...] La enseñanza la coordinamos la moscovita O.N.Filippova y yo (Pokrovskaja, 1989: 59; cita traducida al español del ruso).

La enseñanza en los dos centros es muy intensa: «estudiábamos diecisiete horas consecutivas» (Mihail Ivanov citado en Abramson, 2002: 215), «...hay pausa sólo para comer y un par de minutos de descanso en el jardín donde crecen limones» (Ogievskaja, I967: I88; cita traducida al español del ruso). Sin embargo, el curso en España debe clausurarse «debido a la situación en los frentes» (Mihail Ivanov citado en Abramson, 2002: 215).

En resumen, el GRU se impone como un agente que legitima la práctica de la traducción e interpretación de los traductores y traductoras soviéticos a través de la Jefatura de la Asesoría Militar en diferentes secciones del Ejército Popular de la República. La organización de los centros de formación es otro dato a favor de la institucionalización de la traducción e interpretación en la guerra civil. Todos los agentes involucrados en la traducción, las instituciones, los asesores, los y las intérpretes y los comandantes del ejército popular, poseen a través de la educación un capital cultural y social considerable. Los voluntarios y las voluntarias al pasar la selección reciben un estatus de traductores e intérpretes y empiezan a pertenecer a un grupo seleccionado. El nombramiento oficial de 204 personas como intérpretes militares marca su paso a otro estatus y afirma asimismo su existencia y su valor social como grupo de personas, en la línea de lo que sugiere Bourdieu (I989: 20): «differences function as distinctive signs».

\section{EL DESEMPEÑO DE LOS INTÉRPRETES SOVIÉTICOS EN LA GUERRA CIVIL}

Según las biografías de los y las intérpretes de la URSS (Arcybašev, 2015, T. 2: 294-343), el primer grupo organizado llega a España en octubre de 1936. Los y las intérpretes acompañan a los asesores soviéticos y llevan a efecto, antes que nada, la labor encomendada a estos asesores: «de no haber sido por las traductores e intérpretes nada de lo propuesto por los soviéticos se habría materializado» (Miralles, 2009: 44). Es decir, por medio de la traducción e interpretación posibilitan el trabajo de los asesores en la organización del Ejército Popular de la República (en especial la Marina, la Aviación, la Guerrilla y los Servicios de Inteligencia Militar), en la puesta en funcionamiento de la industria militar, en la organización de las Brigadas Internacionales, en la instrucción y adiestramiento de los voluntarios (Kowalsky, 2004: Chapter 13). De esta manera están presentes en las posiciones estratégicas del ejército republicano.

Desde los primeros días los y las intérpretes encuentran una serie de problemas: tienen que desempeñar una variedad de tareas con las que están poco familiarizados, les falta la práctica comunicativa y, lo principal, desconocen completamente la terminología militar:

[...] sólo en España, al poco tiempo de empezar a trabajar, hemos entendido que además de la traducción en el sentido directo, tendremos que ejecutar una amplia serie de tareas. Resultó que nos tocaba de todo, desde asuntos menos importantes de la vida coti-

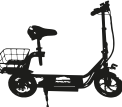

59 
diana hasta problemas más complicados del trabajo de los intérpretes (Fortus, 1967: I8I; cita traducida al español del ruso).

60
[...] me daba miedo hablar con los españoles [...] Resultó que el acento andaluz no era tan diferente del castellano. Los andaluces evitan pronunciar la «d» en la última sílaba, pero en general no era difícil entenderlos (Parshina, 2002: 37).

[...] en el Estado Mayor nos dieron un manual sobre los trabajos de minado, pero muchas palabras que usaba Rodolfo no aparecían allí. [...] No había un diccionario especializado, perdía mucho tiempo en entender, junto con otros soldados, el sentido de las acciones militares... Muchas veces confundía palabras (Starinova, 2015: 5; cita traducida al español del ruso).

Además, los y las intérpretes trabajan en situaciones de alta responsabilidad, tanto en acciones militares como en reuniones del Estado Mayor, subrayada a veces por los propios asesores:

Muchas veces el éxito de las acciones militares o el éxito de uno u otro asesor, sus relaciones con el comando y con los políticos españoles - por cierto de militancia política muy diversa- o a veces incluso la vida del asesor dependían de la formación, de la inteligencia, de la desenvoltura y valor del intérprete (Fortus, 1967: 181; cita traducida al español del ruso).

Si hoy no traduces bien acabaremos todos volando por los aires - me advirtió Artur [Sporgis] (Parshina, 2002: 37).

Los y las intérpretes sirven de mediadores políticos y culturales durante las negociaciones entre las diferentes partes del conflicto. No obstante, la complejidad de la situación política y las verdaderas intenciones de cada una de las partes son solo realmente conocidas por un reducido grupo de acólitos: algunas traductoras e intérpretes comprueban al principio no estar bien enteradas de los conflictos internos. La información sobre la guerra civil española que poseen proviene de medios controlados por el Partido Comunista:

Si en las conversaciones con los artilleros todo era fácil, sincero y claro, en las conversaciones con el subsecretario, que era una persona muy cortés y muy tímida, tuve que aprender a comportarme y a traducir sin emociones. Tuvo que pasar tiempo hasta que aprendí a guardar en secreto mi asombro en cuanto a las contradicciones que reinaban entonces en el Ejército republicano (Ogievskaja, 1967: 188; cita traducida al español del ruso).

En estos encuentros las emociones llegaban a su punto máximo: las traductoras e intérpretes acentúan en sus memorias la necesidad de apaciguar los ímpetus o «intentar dar un matiz tranquilizador» (Parshina, 2002: 52). Como la mayoría del grupo de intérpretes son mujeres llaman a esta estrategia «una censura femenina» (Grinberg, 1989, 245-246), ya que la función de intérpretes es además la de «guardar la imagen del militar soviético» (Samsonova, 1967: 215; cita traducida al español del ruso).

No se trataba sólo de apaciguar los ímpetus, sino a veces, al revés, de dar más expresión a las palabras, hacer que lo dicho resultara más convincente, dotar de más estilo e interés a la frase. Los y las intérpretes establecen la comunicación con los soldados y asumen la tarea educadora y de propaganda ideológica. En esta función se destaca Marija Fortus, la traductora del asesor militar Kirill Mereckov. Así lo recuerda él:

[...] yo empecé mi relato de cómo luchamos en la guerra civil en la Rusia Soviética. Yo hablo y mientras tanto voy observando a la traductora. Marija Aleksandrovna traduce con 
mucho temperamento, la voz tiembla de emoción, la cara se le ha puesto roja. [...] Visto lo larga que resultaba la traducción de mis palabras, Fortus obviamente hacía añadidos, con una entonación muy enérgica y expresiva (Mereckov, 1968: web; cita traducida al español del ruso)

Los y las intérpretes empiezan como acompañantes de los militares, a quienes prestan apoyo personal, psicológico, profesional y militar. A medida que van superando todas las dificultades con la práctica y con un aprendizaje comprometido, en el curso de la guerra van ganando méritos y se convierten en asistentes y combatientes más confiables que los asesores. Desempeñan todo tipo de funciones militares y reciben títulos militares:

Me convertí en una combatiente con los mismos derechos que los otros; yo estaba preparada para compartir el destino con mis amigos (Paršina, I967: 202; cita traducida al español del ruso).

Así, yo, el intérprete, recibí el rango de ayudante del comandante de la submarina C-4 de la Marina del Ejército republicano español y muy pronto recibí el certificado en el que ponían que era teniente de navío (Gurevič, I989: 263; cita traducida al español del ruso).

Con este cambio de estatus, muchas veces los y las intérpretes llegan a imponer sus opiniones y decidir en cuestiones militares. Así, la traductora e intérprete Dar'ja Kravčenko, que trabaja con el general Vicente Rojo, jefe del Estado Mayor del Ejército republicano, comenta sobre el interrogatorio de un piloto extranjero:

Un sexto sentido me decía que era uno de los nuestros. Yo pedí, insistí, supliqué que lo llevasen al hospital y lo conseguí. «Ya tendrán tiempo de ejecutarlo» — dije yo.
Me acerqué al piloto e intenté hablar con él, en ruso, alguien intentó hablarle en alemán, después en francés (Kravčenko, 1967: 223; cita traducida al español del ruso).

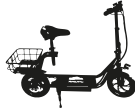

61
La actuación de la intérprete señala su independencia y autoconfianza. Al final no se equivoca: es un piloto soviético, al que encuentra muchos años después ya en la Unión Soviética y quien le agradece que le hubiera salvado la vida. Otro ejemplo ya citado por Rodríguez-Espinosa (2016) es la intervención de Marija Fortus: durante la traducción toma la iniciativa y da órdenes al batallón, animando a los soldados a la lucha (Rodríguez-Espinosa 20I6: 25). Su jefe, el asesor Kirill Mereckov, confirma la capacitación multifacética de Marija Fortus:

[...] resultó capaz de desempeñar no solo las tareas de intérprete, lo que hacía perfectamente, sino también de mantener negociaciones con funcionarios de todos los niveles $y$, al fin y al cabo, ser una oficial digna para cualquier mandato (Mereckov, I968: web; cita traducida al español del ruso)

Analizando estas situaciones, resulta obvia la importancia que adquiere el capital cultural y lingüístico para generar poder y ganar un estatus en la guerra. Este reconocimiento de los y las intérpretes es fruto de un cambio de las categorías de percepción originadas por la situación de la guerra civil, ya que, según Bourdieu (I986), el capital simbólico solo puede generarse dentro de un campo concreto y en relación con los tipos de capital eficientes en él (Bourdieu, r986: 252). La transformación del estatus es todavía más clara en el caso de las mujeres-intérpretes, que a la hora de su selección en Moscú y en los primeros días en la guerra chocan con un prejuicio en relación al género y un rechazo rotundo por parte de los asesores («La guerra no es trabajo para mujeres», en palabras de Vasilij Troján 
en Abramson, 2002: 256). En pocos meses el menosprecio es sustituido por una relación más estrecha y de mutuo apoyo, que finalmente se 62 convierte en un reconocimiento unánime: «Las mujeres intérpretes podrían sustituir a los hombres-asistentes de campo. Saben hablar otras lenguas, son más disciplinadas y responsables, tienen más paciencia y están más atentas en el trabajo» (Grigorij Štern en Pokrovskaja, I989: 6o; cita traducida al español del ruso).

La institucionalización de la traducción e interpretación de los traductores soviéticos sigue con su profesionalización en plena guerra. Se traducen al español todos los materiales de instrucción, los manuales de combate o estrategia y los manuales técnicos de armas. Además del curso de formación de traductores e intérpretes y la asesoría de los jóvenes, se va preparando un diccionario militar terminológico:

Hasta entonces ya tenía un vocabulario bastante sólido de palabras y expresiones de terminología militar general, así como de ingeniería militar (zapadores) y artillería. Otros traductores también llevaban apuntes de términos en diferentes tipos de armas. P.P.Večnyj me propuso encabezar un grupo de trabajo para componer el diccionario. Yo recogía material, lo agrupaba y lo comprobaba. Todos apoyaron la idea del diccionario. $\mathrm{Me}$ llevaba mucho tiempo, era un trabajo adicional, aparte de mis tareas directas. El mismo P.P.Večnyj dedicaba mucho tiempo a redactar «recordatorios» para combatientes y artículos para una revista del frente. Estos materiales también los traducía yo y los llevaba a la redacción (Grinberg, 1989: 244; cita traducida al español del ruso).

La combinación lingüística español-ruso es la que con mayor frecuencia utilizan los traductores e intérpretes soviéticos, pero no la única. Ya que los altos rangos del Ejército español cono-

cían bien el francés y muchos de los y las intérpretes tienen francés como primera lengua, la combinación francés-ruso también fue habitual:

Me alegró especialmente que Uribarry supiera francés y yo podía traducir todo lo que se decía con bastante precisión. Lo que resultó fundamental para establecer una buena relación con el jefe de brigada (Parshina, 2002: 104).

Puesto que faltan especialistas para el italiano, se emplea a las mismas personas para la combinación de lenguas italiano-ruso:

(Interrogatorio de un piloto italiano) El interrogatorio tuve que traducirlo yo. Sin saber ni una palabra de italiano, me preocupaba por la exactitud de la interpretación, pero pronto me tranquilicé. El piloto hablaba una mezcla de español e italiano (Aleksandrovskaja, I986: 246; cita traducida al español del ruso).

El análisis de las situaciones permite comprobar que los y las intérpretes de la URSS dominan las áreas de mando, control e instrucción. Utilizando sus conocimientos culturales y lingüísticos y a través del aprendizaje continuo y comprometido ganan confianza y empiezan a desempeñar profesionalmente una variedad amplia de funciones militares. Usan la traducción para orientarse en el terreno, para ganar información y comunicarla a sus jefes, así como para mediar y obtener control sobre la situación. Los y las intérpretes enviados por el GRU están al servicio de militares soviéticos. Por esta razón, en gran parte de situaciones donde hay asesores involucrados, tienen el ruso como lengua de llegada o de partida.

\section{LOS EFECTOS DE LA INSTITUCIONALIZACIÓN}

La institucionalización de la traducción e interpretación por el GRU en la guerra civil en 
España tiene claros efectos en la práctica de la traducción. Al estar en juego las políticas de diferentes estados y las diversas ideologías surge el problema de la posición que ocupa el traductor e intérprete.

Los y las intérpretes de la URSS pasan un proceso de selección coordinado por los órganos secretos, reciben un nombramiento para la guerra, así como equipamiento e instrucciones para su actuación. Algunas traductoras e intérpretes en sus memorias confirman su ignorancia sobre la complejidad del conflicto (Ogievskaja, I967: 188), y algunas dicen que eran muy jóvenes para poder orientarse en situaciones difíciles o para entender los diferentes tipos de carácter de los seres humanos (Alenikova, 1989: 318). En condiciones de secretismo total, en ocasiones ni siquiera sus familias saben dónde están (Zernova, 1998: 136), vienen a cumplir una misión asignada por el GRU:

En lo que atañe a la guerra civil en España, hasta los años 60 estaba prohibido mencionar en público la participación soviética en ella, todos los voluntarios habían estado en una comisión de servicio. Al buen entendedor con pocas palabras basta (Abramson, 2002: 188).

El proceso de institucionalización, empezando por la educación, los medios de comunicación y el entorno social, va guiando la percepción del mundo y la actuación de las personas. «Nos recomendaron que tanto al partir como al regresar no lleváramos libreta de apuntes, notas, números de teléfono, direcciones, fotos, etc.... En aquellos años, la mayoría de los soviéticos cumplía a pies juntillas las recomendaciones», señala Mihail Ivanov, uno de los intérpretes en España (citado en Abramson, 2002: 215). E1 grupo soviético recibe estas y otras recomendaciones ya en Moscú: «Antes de mi partida, el camarada Vorošílov me dio unas breves instruc- ciones con respecto al trabajo de nuestra gente», confirma el segundo Asesor Jefe, Grigorij Štern (Miralles, 2009: 34). Asimismo, muchas veces se dan órdenes desde Moscú de cuál debe ser el orden de las operaciones (Miralles, 2009: 34).

Estos datos nos llevan a la conclusión de que, tanto los asesores, como los y las intérpretes disfrutan de poca autonomía de actuación profesional y dan curso a la política del estado. $\mathrm{Su}$ deseo de luchar contra el fascismo y por la solidaridad internacional (Batov, I965: 286; Grinberg, 1989: 236) se ve instrumentalizado por los órganos secretos del Ejército Rojo. Según Bourdieu, es un caso de violencia simbólica y «of that monopoly over legitimate symbolic violence which belongs to the state or to its representatives» (Bourdieu, I989: 2I). Las personas, aunque involuntariamente, se convierten en instrumentos del poder y ponen a favor de los intereses del estado su actividad profesional, que en el caso de los intérpretes es la práctica de la traducción e interpretación.

Otro aspecto de la institucionalización es el de la protección y estabilidad que proporciona a sus agentes. Según recuerda Adelina Abramson:

A los que dependíamos del GRU, el NKVD no nos gustaba demasiado. Incluso diría que entre ambos organismos hubo una verdadera competencia. Desde luego, la gente del GRU era mucho más culta y preparada que «nuestros vecinos» (así llamábamos nosotros, coloquialmente, a la gente del NKVD). [...] A Alexander Orlov, del que tanto se ha hablado, no lo conocí, pero creo que fue una de las personas de más negro rastro que hubo en España [...] (Abramson, 2009: 137)

La autora habla con confianza sobre el GRU y con cierto desprecio sobre el NKVD, la organización más temible en España y cuya misión fue «la de reprimir la disidencia en el campo republicano o, dicho de otro modo, extender las 
purgas estalinistas a España» (Miralles, 2009: 28). A partir del ejemplo de su hermana Paulina Abramson subraya una cierta autonomía y libertad de decisión para quienes traducen:

A mi hermana Lina, que llevaba en España desde octubre de 1938 , y que incluso había ingresado como militante del Partido Comunista Español, la mandaron para que hiciera de intérprete de Orlov. Este le hizo un interrogatorio bastante desagradable, lo cual causó tan mala impresión a Lina que se negó en rotundo a trabajar con él (Abramson, 2009: 137).

Los y las intérpretes que acompañaban a los asesores desarrollan una relación de cercanía y de confianza con sus jefes. El apoyo mutuo, lingüístico y psicológico, en situaciones de riesgo contribuye a formar nexos para toda la vida, algunos incluso de orden familiar: Aleksandrovskaja-Ptuhin, Obručeva-Starinov, Abramson-Mamsurov, Karmen-Konstantinovskaja, Paršina-Sporgis, etc. Al servicio de los asesores o de los comandantes soviéticos tienen unas condiciones de vida privilegiadas: residen en casas separadas (en Albacete en la c/ de San Antonio, 3er piso, Botin, 1976: 44) o en hoteles (en Barcelona en el hotel «Diagonal», Gurevič, I989: 258). Gracias a las redes sociales que forman en la universidad hay un clima de confianza, seguridad y camaradería. En la guerra se establece un protocolo de asesoría a los jóvenes antes del relevo de turno. Su evacuación de España está también planeada; tienen todos los documentos necesarios para cruzar las fronteras y el transporte organizado (Plavskin, I989: 337).

La institucionalización de la traducción e interpretación en la guerra civil española resulta ser una plataforma de formación de un grupo de traductores militares que al volver de España siguen formándose profesionalmente en la
Academia Militar Frunze (Paulina Abramson, Nora Čegodaeva, Marija Fortus, Ljubov' Meston, Elizaveta Paršina, etc.). Aparte, hay que mencionar su aportación en el desarrollo de la profesión de «intérprete militar». Después de la guerra civil española, el gobierno de la Unión Soviética intenta solventar la falta de militares con conocimientos de lenguas y en I940 se funda el Instituto Militar de Idiomas del Ejército Soviético, donde, por ejemplo, enseñan español Paulina Abramson, Adelina Abramson, Aleksej Korobicin y otros intérpretes de la guerra civil. La mayoría de los intérpretes de la guerra civil participa en la Segunda Guerra Mundial, donde algunos de ellos dan sus vidas (Ljudmila Černik, David Frankfurt, Semën Gankin, Vladimir Grigor'ev, Mihail Makarov, Solomon Solovejčik). Al acabar las guerras vuelven a las universidades, hacen carrera académica y trabajan en el ámbito de la traductología o la traducción literaria del español al ruso (Nina Butyrina, Valerij Stolbov, Georgij Stepanov, Ruf Zernova, Ivan Trista-Sokolov).

\section{CONCLUSIONES}

La institucionalización es un proceso mediante el cual se establecen ciertas prácticas habituales en la vida social, tales como la traducción e interpretación que fueron ejecutadas por el grupo soviético de intérpretes en la guerra civil. El nombramiento para la guerra de los 204 intérpretes queda documentado y archivado en el Archivo Militar de la Federación Rusa (RgvA, F.33987. I.3.A.II43. P.r27). Gracias a estos documentos y a las biografías publicadas (Arcybašev, 20I5, T. 2: 294-343) sus nombres han sido rescatados del olvido.

A través del mecanismo de la institucionalización, los y las intérpretes reciben un estatus concedido por el GRU y van ganando impor- 
tancia y reconocimiento social en función de su desempeño en la guerra. Acompañando a los asesores soviéticos, están en todos los puntos clave del Ejército republicano y desempeñan una gran variedad de funciones. La institucionalización proporciona una plataforma para obtener competencias, conocimientos y habilidades profesionales. Las redes sociales y profesionales se forman dentro de los centros educativos o en los puestos de trabajo. Al final, la traducción e interpretación en muchas áreas de la guerra civil española se convierte en una práctica planeada y coordinada por una institución, que trata de alcanzar así sus intereses propios.

La institucionalización de una práctica social en los regímenes totalitarios adquiere muchas veces un carácter forzoso que convierte tanto a las personas como las ideas en herramientas de poder que de manera irracional o, algunas veces, privadas del derecho a razonar los lleva a manipular ideas a favor de la ideología dominante. Así, la institucionalización origina dos tendencias al mismo tiempo: por un lado, los traductores e intérpretes reciben apoyo institucional; por otro lado, se someten al cumplimiento de las normas propias de la institución.

RECIBIDO EN AGOSTO DE $20 I 8$ ACEPTADO EN FEBRERO DE 2019 VERSIÓN FINAL DE JUNIO DE 2019

\section{FUENTES AUTOBIOGRÁFICAS}

Abramson, Adelina (2009): «Yo estuve en España», en VV.AA., Los rusos en la guerra de España, 19361939, Madrid: Fundación Pablo Iglesias, I35-I38.

Abramson, Paulina y Adelina Abramson (2002): Mosaico roto. Madrid: Compañía Literaria.

Aleksandrovs kaja, Sof ja (I986): "Takimi ja ih pomnju», en Sof ja AleKsandrovskaja (ed.), $M y$ - internacionalisty: vospominanija sov. dobrovol'cev
- učastnikov nac.-revoljuc. vojny v Ispanii. Moscú: Politizdat, $242-258$.

Alenikova, Ėsfir‘ (I989): «Ispanija dalekaja i blizkaja», en Anatolij GUREVIČ y David PRICKER, (eds.), Leningradcy v Ispanii, Leningrado: Lenizdat, 318-333.

BAtov, Pavel (I965): «V rjadah dobrovol'cev svobody», en Nikolaj VORONOV (ed.), Pod znamenem Ispanskoj respubliki. 1936-I939: Vospominanija sov. dobrovol'cev-ućastnikov nac.-rev. vojny v Ispanii, Moscú: Nauka, 46-70.

Botin, Mihail (1976): $S$ toboj, Ispanija, Moscú: Voenizdat.

Dufek, Karel (2008): «Soudržnost interbrigadistů», en Memoria de las Naciones. http://www.pametnaroda.cz/witness/clip/id/42/clip/32 [consulta: I5-II-20I9].

Eisner, Alexei (I972): La XII Brigada Internacional, trad. Arnaldo Azzati, Valencia: Edit. Prometeo.

Èjsner, Aleksej (I986): Čelovek s tremja imenami: Povest o Matè Zalka, Moscú: Politizdat. https:// www.e-reading.club/chapter.php/IoI5623/2/Eysner_Chelovek_s_tremya_imenami_Povest_o_ Mate_Zalke.html [consulta: I-VI-20I8].

Fortus, Marija (I967): "Ne tol'ko perevodčiki», Znamja, 3, I80-187.

Grinberg, Genrietta (1989): «V Aragone i Katalonii», en Anatolij Gurevič y David Pricker, (eds.), Leningradcy v Ispanii, Leningrado: Lenizdat, $235-248$.

Gurevič, Anatolij (I989): «Na podvodnoj lodke čerez Gibraltar», en Anatolij Gurevič y David Pricker, (eds.), Leningradcy v Ispanii, Leningrado: Lenizdat, 254-290.

KravčEnko, Dar'ja (I967): «Trudnye dni. Ishod», Znamja, 3, 222-226.

Mereckov, Kirill (i968): Na službe narodu, Moscú: Politizdat.

Ogievskaja, Evgenija (i967): «Na vsju žizn'...», Znamja, 3, I88-I89.

Parshina, Elizaveta (2002): La Brigadista. Diario de una dinamitera de la Guerra Civil, trad. Dimitri Fernández Bobrovski, Málaga: La Esfera de los Libros.

PArshina, Elizaveta (i967): «V vojskovoj razvedke», Znamja, 3, 200-203.

Plavskin, Zaharij (I989): «Poslednie dni respubliki», en Anatolij Gurevič y David Pricker, (eds.), Leningradcy v Ispanii, Leningrado: Lenizdat, 333-354.

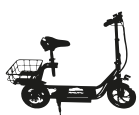

65 


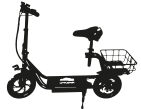

66
Pokrovskaja, Ljusija (I989): "Glazami perevodčicy», en Anatolij Gurevič y David Pricker, (eds.), Leningradcy v Ispanii, Leningrado: Lenizdat, 48-62. Pricker, David (I989): «Ispanija, uvidennaja triždy», en Anatolij Gurevič y David Pricker, (eds.), Leningradcy $v$ Ispanii, Leningrado: Lenizdat, 354-390.

Samsonova, Ljubov' (1967): «Pobedy i poraženija», Znamja, 3, 213-222.

Starinova, Anna (2015): Naša sojuznica noč. Moscú: Algoritm. http://litread.in/pages/558807/58600o58700o?page $=$ I [consulta: $15-\mathrm{II}-2019$ ].

Zernova, Ruf' (i998): Na more i obratno. Jerusalem: URA Publishers.

\section{REFERENCIAS BIBLIOGRÁFICAS}

Arcybašev Valerij, Oleg Karimov y Igor' VološEnko (eds.). (20I5): Iz Moskvy - v stranu «Iks». Kniga pamjati sovetskih dobrovol'cev učastnikov Graždanskoj vojny v Ispanii 1936-I939 gg, Moscú: Glavnoe arhivnoe upravlenie g. Moskvy. Central'nyj gos. arhiv g. Moskvy.

BAigorri Jalón, Jesús (2OI2): «La lengua como arma: intérpretes en la guerra civil española o la enmarañada madeja de la geografía y la historia», en Gertrudis Payàs y José Manuel Zavala (eds.), La mediación lingüístico-cultural en tiempos de guerra: cruce de miradas desde España y América, Temuco, Chile: Universidad Católica de Temuco, 85 -108.

Bourdieu, Pierre (1986): «The Forms of Capital», en John Richardson (ed.), Handbook of Theory and Research for the Sociology of Education, New York: Greenwood, 24I-258.

Bourdieu, Pierre (I989): «Social Space and Symbolic Power», Sociological Theory, 7 I I (primavera), I4-25. http://www.jstor.org/stable/20206o [consulta: I-VI-2018].

Bourdieu, Pierre (1993): Sociology in Question, trad. Richard Nice, Londres: Sage.

Brigadistas: el archivo fotográfico del General Walter (2005): [textos, Jesús González de Miguel, Gabriel Jackson, Ángel Rojo Rincón]. Madrid: Ayuntamiento, Área de Las Artes [etc.], 2005.
Hermans, Theo (I995): «Translation as Institution», en Mary Snell-Hornby, Zuzana Jettmarová y Klaus Kaindu (eds.), Translation as Intercultural Communication: Selected papers from the EST Congress, Ámsterdam: John Benjamins, 3-20.

Inghilleri, Moira (2005): "The Sociology of Bourdieu and the Construction of the 'Object' in Translation and Interpreting Studies», The Translator, $\mathrm{II} / 2, \mathrm{I} 25^{-\mathrm{I}} 45$.

Inghilleri, Moira y Sue-Ann Harding (20Io): "Translating Violent Conflict», The Translator, I6/2, I65-I73.

KANG, Ji-Hae (1998): "Institutional translation», en Mona Baker (ed.), Routledge Encyclopedia of Translation Studies. Londres: Routledge.

Keman, Hans (20I7): «Institutionalization: social process», en Encyclopadia Britannica. https:// www.britannica.com/topic/institutionalization [consulta: I-VI-2018].

Kirschenbaum, Lisa A. (2018): International communism and the Spanish Civil War, Cambridge: Cambridge University Press.

Kowalsky, Daniel (2004): Stalin and the Spanish Civil War, Nueva York: Columbia University Press. http://www.gutenberg-e.org/kodor/frames/fkodimg.html [consulta: I-VI-20I8].

Miralles, Ricardo (2009): «Los soviéticos en España, 1936-1939", en VV.AA., Los rusos en la guerra de España, I936-I939, Madrid: Fundación Pablo Iglesias, $18-47$.

Rybalkin, Yuri (2009): "La ayuda militar soviética a la República español. Cifras y hechos», en VV.AA., Los rusos en la guerra de España, I9361939, Madrid: Fundación Pablo Iglesias 66-8I.

Rodríguez-Espinosa, Marcos (20I6): "No Pasarán!'. Translators Under Siege and Ideological Control in the Spanish Civil War", Perspectives. Studies in Translatology, 24 I, 22-35.

Wolf, Michaela (20I7): "Hundert Sprachen, doch ein Wille': Dolmetschen bei den Internationalen Brigaden», en Georg Pichler y Heimo HaLBrainer (eds.), Camaradas. Österreicherinnen und Österreicher im Spanischen Bürgerkrieg I936-I939, Graz: Clio, I53-I68.

Wolf, Michaela y Alexandra Fukari (2007): Constructing a Sociology of Translation. Ámsterdam/ Filadelfia: John Benjamins Publishing. 